\title{
Authentification et légitimation d'un problème de société par les journalistes : les violences de genre en banlieue dans la médiatisation de Ni putes ni soumises
}

Authentication and Legitimization of a Social Issue by Journalists: Gender-

Related Violence in the French Suburbs in the Media Coverage of Ni Putes ni

Soumises

Marion Dalibert

\section{OpenEdition}

\section{Journals}

Édition électronique

URL : http://journals.openedition.org/edc/5214

DOI : 10.4000/edc.5214

ISSN : 2101-0366

Éditeur

Université Lille-3

Édition imprimée

Date de publication : 1 juin 2013

Pagination : 167-180

ISBN : 978-2-917562-09-3

ISSN : 1270-6841

\section{Référence électronique}

Marion Dalibert, «Authentification et légitimation d'un problème de société par les journalistes : les violences de genre en banlieue dans la médiatisation de Ni putes ni soumises », Études de communication [En ligne], 40 | 2013, mis en ligne le 01 juin 2013, consulté le 21 avril 2019. URL : http:// journals.openedition.org/edc/5214; DOI : 10.4000/edc.5214

Ce document a été généré automatiquement le 21 avril 2019

(c) Tous droits réservés 


\title{
Authentification et légitimation d'un problème de société par les journalistes : les violences de genre en banlieue dans la médiatisation de Ni putes ni soumises
}

\author{
Authentication and Legitimization of a Social Issue by Journalists: Gender- \\ Related Violence in the French Suburbs in the Media Coverage of Ni Putes ni \\ Soumises
}

Marion Dalibert

1 Un mouvement social se crée après avoir identifié un problème de société, et son objectif est, notamment, de mobiliser la sphère politique afin que celle-ci envisage les mesures pour le prendre en charge. Il est donc contraint de lutter pour la visibilité (Voirol, 2005) et de mettre en place des stratégies pour atteindre les médias d'information généraliste (Molotch et Lester, 1996), ces derniers ayant un rôle d'intermédiaire entre les pouvoirs publics et l'action collective (Neveu, 1999). En permettant à un problème de société d'atteindre la visibilité sociale (Arendt, 1961; Thompson, 2005), les journalistes procèdent à des opérations de cadrage qui peuvent conduire à le légitimer, auprès des publics et des politiques, en le construisant comme étant «réel». Grâce à ce processus d'authentification corrélé à la médiatisation (et à une médiatisation conséquente), la sphère politique est généralement contrainte de répondre à la cause d'un groupe protestataire. La couverture médiatique de Ni putes ni soumises (NPNS), mouvement social né en janvier 2002 pour dénoncer l'augmentation des violences de genre à l'intérieur des banlieues françaises, est, de ce point de vue, exemplaire.

2 Après quatorze mois de mobilisation, le collectif a accédé aux agendas médiatique et politique lors de la clôture, à l'occasion de la Journée internationale des femmes, de sa 
«Marche des femmes des quartiers pour l'égalité et contre le ghettos » qui a eu lieu du $1^{\mathrm{er}}$ février au 8 mars 2003 dans toute la France. La dernière étape de cette action collective a été "promue au rang d'événement» médiatique (Krieg, 1996, 114-115) et la cause de NPNS a été prise en charge par le gouvernement: les médias ont couvert de manière importante (et valorisée) le groupe protestataire ${ }^{1}$, et le Premier ministre Jean-Pierre Raffarin a reçu les membres du mouvement et annoncé des mesures à mettre en place pour lutter contre le sexisme en banlieue. Avant ce rendez-vous à Matignon, le problème de société porté par NPNS a été authentifié, dès la naissance de la mobilisation, par et au sein des médias d'information généraliste.

3 Le cadrage des articles et des séquences de journaux télévisés (JT) n'a pas nécessairement porté sur le mouvement lui-même ou sur les actions mises en place (diffusion de l'Appel et du Manifeste des femmes des quartiers, organisation d'états généraux...), c'est le quotidien des jeunes filles à l'attribut ethnoracial marqué ${ }^{2}$ qui a occupé l'espace médiatique. Les journalistes ont uniquement mis en scène une victime collective, les adolescentes et jeunes femmes "non-blanches", face à un coupable, les «jeunes garçons des banlieues ». Ils n'ont cependant jamais fait état de la mise en cause, par NPNS, des politiques publiques successives qui ont isolé et précarisé les habitants des quartiers populaires et qui ont conduit, selon le mouvement, au développement des violences de genre. Le groupe protestataire ne souhaitait pas opposer les femmes et les hommes vivant dans les banlieues, il voulait que les individus des deux genres rejoignent leur combat contre l'État, ce dernier étant désigné comme le vrai coupable du sexisme étant donné qu'il n'a pas réagi à l'augmentation des discriminations commises à l'encontre des habitants de cette zone géographique ethnoracialisée (Hancock, 2008). Le cadrage opéré par les journalistes a réactivé les représentations et "discours sociaux " (Delforce et Noyer, 1999) portant sur la banlieue et les minorités ethnoraciales qui circulent dans les médias depuis les années 1980 : un jeune garçon d'origine maghrébine, stéréotype négatif $\mathrm{du}$ " jeune des banlieues », a été mis en scène comme agressant (souvent sexuellement) sa sœur et celles de ses copains ${ }^{3}$.

4 Cette analyse est basée sur l'étude, effectuée dans une démarche constructiviste d'analyse de discours, de la médiatisation de NPNS de janvier 2002 à mars 2003 dans la presse quotidienne nationale dite « de référence » (le Figaro, le Monde et Libération) et dans les JT de TF1, France 2 et France 3. En totalité, le corpus comprend 33 articles et 16 sujets de JT ${ }^{4}$.

\section{La fonction authentifiante du témoignage}

5 Les médias d'information ont un statut ou, devrait-on dire, une autorité particulière à l'égard de la «réalité »: ils sont censés garantir à leurs publics qu'ils reproduisent fidèlement et en toute neutralité la "réalité » du monde. Cependant, le «discours d'information » (Charaudeau, 2005) est performatif ${ }^{5}$ : il produit des savoirs qu'il transmet comme étant réels, il ne les reproduit pas (Quéré, 1982, 158). Il ne fait pas que décrire ce qui se passe dans le monde. Il produit, en même temps, la réalité sociale du monde.

6 Ce processus de construction de la réalité dépend néanmoins des genres des articles et des sujets de JT, ces derniers n'étant pas pourvus de la même légitimité face à la construction sociale de la réalité, attendu qu'ils ne sont pas dotés du même « contrat de communication » (Charaudeau, op. cit.), contrat qui permet à l'« instance de production » et à l'« instance de réception-interprétation » d'identifier la situation de communication dans laquelle ils sont investis et d'agir en fonction de ses contraintes et modalités (ibid., 
52). Le lecteur d'un quotidien et un téléspectateur de JT ne recevront pas un compte rendu de la même manière qu'une critique de film, car leur "visée " (ibid.) - ou leur « promesse » vis-à-vis de la réalité (Jost, 2003, 19) - est dissemblable. Dans le premier cas, la visée est informative - et performative -, dans le second cas, elle est plus persuasive.

De janvier 2002 à mars 2003, et à l'exception d'un éditorial publié dans le Monde en octobre 2002, les genres utilisés pour rendre compte de NPNS ont tous une visée informative (synthèses, reportages, témoignages ${ }^{6}$, enquêtes, comptes rendus...). En faisant la promesse à l'instance de réception-interprétation que ce qui est relaté au sein des JT et des quotidiens est effectivement « réel », les genres ont authentifié le problème de société du sexisme en banlieue.

Les journalistes se sont déplacés dans les quartiers populaires de la périphérie de Paris pour y interroger des adolescentes «non-blanches » au sujet de leurs rapports entretenus avec leur famille et les garçons de leur âge, et leur ont demandé de se raconter, de dire qui elles étaient et de décrire leur vie quotidienne. Cette collecte de témoignages renvoie à la procédure de l'aveu, décrite par Foucault, pour produire la vérité sur le sexe (Foucault, 1976). En faisant parler ces adolescentes sur elles-mêmes, en rendant visible leurs paroles au sein de genres aux visées informative et performative, le «type » " adolescente 'nonblanche' qui vit en France » a été créé comme « réel ». Il a été authentifié. Pour Foucault, «l'aveu est devenu, en Occident, une des techniques les plus hautement valorisées pour produire le vrai [...]. L'individu s'est longtemps authentifié par la référence des autres et la manifestation de son lien à autrui (famille, allégeance, protection); puis on l'a authentifié par le discours de vérité qu'il était capable ou obligé de tenir sur lui-même » ( ibid., 78-79).

9 Les adolescentes interrogées ont fait office de "sujets d'énonciation théorique » (Jost, op. cit., 65): chacune d'entre elle a incarné un "témoin " représentatif d'un groupe partageant les mêmes attributs catégoriels (genre, âge, attribut ethnoracial, classe sociale), vie quotidienne et difficultés. Ce groupe a été catégorisé : les journalistes ont représenté et construit un groupe homogène qui partage la même identité sociale, les mêmes rapports avec l'entourage, le même caractère, les mêmes souffrances. Les adolescentes qui ont témoigné ont été décrites et mises en scène en tant que "nonblanches » alors qu'au départ, les membres de NPNS se sont mobilisés à la fois pour les femmes «blanches" et "non-blanches" appartenant aux classes populaires. Or, le groupe pour qui le collectif a formulé des revendications s'est incarné médiatiquement dans le stéréotype de la «jeune fille des banlieues», adolescente ou jeune femme d'origine maghrébine de nationalité française.

Les «jeunes filles des banlieues» ont été ethnoracialisées ${ }^{7}$ : une frontière entre les «blancs» et les «non-blancs» a été produite discursivement. La stéréotypisation, la catégorisation et l'ethnoracialisation sont ici des synonymes: ils renvoient à un même processus de différenciation des groupes sociaux. Chaque témoignage a contribué à la catégorisation du groupe des jeunes filles "non-blanches", groupe qui n'a d'existence médiatique qu'en fonction de l'imbrication de son genre et de son attribut ethnoracial. 


\section{La valorisation du discours rapporté des « jeunes filles des banlieues »}

11 Les témoignages des jeunes femmes à l'attribut ethnoracial marqué, valorisés par l'utilisation de la citation dans la presse écrite et de l'interview dans les JT, ont participé à l'authentification du stéréotype féminin construit dans la médiatisation de NPNS ${ }^{8}$. Les institutions médiatiques ont toutes mis en avant le discours rapporté des militantes du groupe protestataire et celui de jeunes filles vivant dans des quartiers populaires à la périphérie de Paris. La presse a promu les propos d'adolescentes «non-blanches", comme dans le Monde: "'Un jour, mon cousin m'a baffée parce qu'il avait trouvé des cigarettes dans mes affaires', raconte Kahina. 'Lui, il fume depuis l'âge de 13 ans, mais moi,je n'ai pas le droit. Ce n'est pas pour ma santé, c'est parce qu'il trouve que c'est vulgaire' "'. Et la télévision a diffusé de nombreuses interviews de jeunes filles témoins, comme sur France 3: «On sait plus vraiment qu'elle est la voie à choisir, parce qu'il y a à la fois la vie à l'intérieur du quartier où on est complètement cachées! C'est le terme ouais ! On est complètement cachées [...]! Donc au bout d'un moment, on peut plus quoi ! $»^{10}$.

12 L'étude du paradigme désignationnel ${ }^{11}$ (Mortureux, 1993) du groupe pour qui NPNS s'est mobilisé a montré que, de janvier 2002 à mars 2003, les militantes du mouvement et les jeunes femmes interrogées ont été auteures de plus du tiers des syntagmes utilisés pour désigner la figure féminine victime de violences de genre (tableau 1).

Tableau 1 - Les auteurs des syntagmes désignatifs ayant pour référent les adolescentes «nonblanches » (de janvier 2002 à mars 2003)

\begin{tabular}{|l|l|l|l|l|}
\hline & Journalistes & $\begin{array}{l}\text { Membres } \\
\text { de NPNS }\end{array}$ & $\begin{array}{l}\text { Jeunes filles } \\
\text { interrogées dans } \\
\text { les banlieues }\end{array}$ & $\begin{array}{l}\text { Autres (infirmières scolaires, } \\
\text { responsables associatifs, jeunes } \\
\text { garçons interrogés dans les } \\
\text { banlieues...) }\end{array}$ \\
\hline $\begin{array}{l}\text { Nombre de } \\
\text { syntagmes } \\
\text { désignatifs }\end{array}$ & 290 & 135 & 53 & 43 \\
\hline Pourcentage & $56 \%$ & $26 \%$ & $10 \%$ & $8 \%$ \\
\hline
\end{tabular}

13 Les journalistes, qui ne portent qu'un peu plus de la moitié des reformulants relevés dans le corpus (tableau 1), ont mis en avant les paroles d'individus faisant autorité, par rapport à l'imbrication de leurs attributs catégoriels (genre et attribut ethnoracial), dans la production du savoir entourant les adolescentes qui vivent dans les banlieues : celles des militantes du mouvement et des jeunes filles «non-blanches ».

14 De janvier 2002 à mars 2003, les membres de NPNS ont été mises en scène comme des sujets d'énonciation théorique; elles n'ont pas été présentées et décrites comme des militantes d'une organisation, mais uniquement comme des jeunes femmes à l'attribut ethnoracial marqué. Le collectif protestataire et le groupe pour qui il s'est mobilisé se sont confondus. À l'image des adolescentes interrogées par les journalistes, les membres de NPNS sont désignés uniquement par leur prénom ("Safia », «Loubna », "Christelle »...). Comme elle dispose d'un statut de responsable associative à l'égard des médias, Fadela 
Amara (présidente de la Fédération nationale des Maisons des potes jusqu'en mars 2003) a fait figure d'exception : son nom de famille est précisé à chaque fois.

Amara et les autres militantes de NPNS ont été mises en scène en tant que femmes ethnoracialisées. Leur biographie est «typique » des femmes d'origine maghrébine. Alors que le mouvement comptait à la fois des militantes «blanches » (Christelle Raspolini et Ingrid Renaudin) et «non-blanches $»^{12}$ (Fadela Amara, Safia Lebdi et Loubna Méliane), ces dernières ont été beaucoup plus interrogées par les journalistes. De janvier 2002 à mars 2003, $89 \%$ des militantes interviewées ou citées dans le corpus sont d'origine maghrébine ${ }^{13}$. En outre, celles qui ont fait l'objet d'un article ou d'un sujet de JT (du genre du portrait ou de l'interview) ont toujours été « non-blanches ».

Dans une interview de Safia Lebdi publiée dans Libération le 8 mars 2003, celle-ci incarne le stéréotype de la «jeune fille des banlieues». Son histoire n'est pas présentée par le journaliste (dans le paragraphe introductif) comme étant celle «type» de la femme occidentale : Lebdi vient d'une famille particulière à la culture spécifique : «Safia [...] a 29 ans, vient de Clermont-Ferrand. Sa famille 'ressemble à n'importe quelle famille maghrébine, avec ses traditions et sa culture'. Petite dernière à la maison, elle a dû se battre $»^{14}$.

En février 2003, Libération ${ }^{15}$ et le Figaro ${ }^{16}$ ont chacun publié un portrait de Fadela Amara. La future présidente de NPNS y est principalement décrite comme une femme à l'attribut ethnoracial marqué : elle est d'origine nord-africaine, musulmane pratiquante, elle a vécu dans un quartier populaire de Clermont-Ferrand et a été élevée au sein d'une famille avec de nombreux frères et sœurs, un père travailleur immigré et une mère au foyer qui a rejoint son mari lors de la politique du regroupement familial dans les années 1970. Parce qu'elle partage le genre et l'attribut ethnoracial du groupe social dont elle veut défendre les intérêts, Amara a été mise en scène comme le témoin privilégié (et authentique) de la dégradation des rapports de genre dans les banlieues et de la régression du statut de la femme «non-blanche» en France. Son histoire est mise en avant comme caution d'authenticité à la fois dans le Figaro :

Pour conter la désagrégation des cités, Fadela n'a pas de chiffres, juste « des témoignages, du vécu et du recul ». Parce qu'elle est née à Herbet, une cité d'urgence de Clermont-Ferrand. Elle grandit dans une famille de dix enfants, père kabyle, ouvrier dans le bâtiment, mère au foyer

et dans Libération:

Fadela Amara est la fille d'une cité de Clermont-Ferrand. Dans ce quartier "aux murs délabrés, où tout transpirait la pauvreté », les adolescents aidaient les vieilles à porter leurs courses, et tout le monde rappliquait quand le malheur frappait une famille [...]. Fadela Amara a repéré les dégâts en bas de chez elle. «Des filles me disaient : 'Mon frère ne veut pas que j'aille en boîte', ou 'Je n'ai pas le droit d'aller en fac à Toulouse', et elles s'inclinaient » [...]. Constat particulièrement cruel puisque, comme beaucoup d'autres femmes nées dans les années 1960 de parents immigrés, Fadela était partie à la conquête de sa liberté. Et, sans jamais rompre avec sa famille, l'avait finalement arrachée.

L'identité sociale d'Amara fait valeur d'argument dans la production de la réalité du problème de société du sexisme en banlieue. Elle sait parce qu'elle est. La mise en scène médiatique de ses attributs identitaires et de sa biographie a pour dessein d'authentifier et de légitimer la cause du mouvement auprès des publics. 


\section{Le stéréotype de la « jeune fille des banlieues »} similaires d'une institution médiatique à l'autre. Le produit de ces discours, le stéréotype de la "jeune fille des banlieues", a été catégorisé par son genre. Caractérisé par sa féminité, il a été mis en scène comme victime du sexisme des adolescents masculins «non-blancs ». Les « jeunes filles des banlieues » sont dépeintes comme étant exposées à des violences verbales récurrentes (elles doivent faire face aux insultes quotidiennes de " putes » et de «salopes ») et comme étant contraintes de rester vierges pour protéger leur réputation, comme l'authentifie, par exemple, le discours rapporté de «Sarah » dans le Monde: « Par réflexe d'autodéfense, elles résistent à la demande sexuelle des garçons, car perdre sa virginité, c'est perdre sa réputation. 'Il ne faut pas se donner comme ça, il ne faut pas se salir et faire complètement confiance à quelqu'un', explique Sarah $»^{17}$.

21 En plus des injures, elles sont décrites comme étant surveillées par leurs frères et comme étant battues s'ils les jugent trop émancipées. Dans Libération, «Karima » a été invitée à rendre compte des violences reçues par son frère lorsqu'il a appris qu'elle possédait un string:

\footnotetext{
Est-ce qu'elles fument? Sortent? Comment s'habillent-elles? Qui fréquentent-elles? Les frères veillent. Karima, 24 ans, rapporte ainsi comment a commencé chez elle, à Clermont-Ferrand, ce qu'elle intitule la " guerre du string ». Pour son anniversaire, des copines lui offrent un string. Son frère le trouve par hasard quelques semaines plus tard, «il rentre dans une colère monstre, j'ai dû me justifier en lui donnant des explications, puis j'ai reçu des coups, sans compter les insultes $»^{18}$.
}

Les jeunes filles «non-blanches" sont construites dans le discours d'information comme risquant quotidiennement d'être violées par un ou plusieurs garçons de leur entourage souvent très proche (un voisin, le frère, un petit ami). France 2 a par exemple diffusé l'interview d'une adolescente témoin qui a fait état du chantage dont font l'objet les filles victimes de viols collectifs, chantage qui les oblige à se soumettre aux désirs des garçons :

Si y'a une quinzaine de mecs - même pas - cinq mecs qui viennent vers vous et qui vous entraînent à un endroit et qui vous font du chantage parce que c'est souvent comme ça ! C'est ça qu'on dit pas! Y'a du chantage vis-à-vis de la famille, des frères, des sœurs! De tout ça! Donc la personne elle est obligée de se taire parce qu'elle a peur des représailles de sa famille ${ }^{19}$.

Dans la médiatisation de NPNS, les femmes et hommes «blancs » n'ont pas été mis en cause, que cela soit en tant que victimes ou en tant que coupables. Les rapports asymétriques de genre ont été construits comme étant propres aux minorités ethnoraciales. La violence des garçons «non-blancs» est décrite comme étant une réponse (et surtout une punition) à la «féminité » et à l'«émancipation» des adolescentes à l'attribut ethnoracial marqué : c'est lorsqu'elles adoptent les normes occidentales de genre qu'elles subissent des sévices. Dans le Figaro, Fadela Amara a expliqué que «les femmes doivent raser les murs, se faire transparentes, nier leur féminité, pour éviter les ennuis $\aleph^{20}$. Un présentateur du JT de $20 \mathrm{~h}$ de France 3 a introduit un sujet en décrivant les femmes d'origine maghrébine comme étant contraintes par leur culture dans leurs demandes de liberté: "Voici l'exemple de trois jeunes femmes issues de 
l'immigration, elles habitent à Asnières en Région Parisienne et supportent mal leur condition, écartelées entre une tradition pesante et leur volonté d'émancipation ${ }^{21}$.

Les violences que subissent les adolescentes «non-blanches » sont représentées comme étant d'une brutalité exceptionnelle - dont les femmes «blanches » ne sont, a priori, pas victimes. Libération a rapporté le discours de Safia Lebdi où elle raconte que «l'une de [s]es copines a été égorgée devant ses enfants parce qu'elle voulait divorcer $"{ }^{22}$. Certes, les femmes occidentales subissent des violences, mais celles qui sont supportées par les femmes à l'attribut ethnoracial marqué sont représentées, dans les médias, comme étant quotidiennes et plus cruelles. Les "jeunes filles des banlieues" sont mises en scène comme risquant de subir un viol collectif alors que pour les femmes occidentales, il s'agira d'un "simple» viol. Les jeunes femmes «blanches" appartenant aux classes populaires ont quelques fois été décrites comme étant victimes de violences, mais l'oppression est présentée comme plus occasionnelle et moins importante, comme l'illustre cet extrait d'un article de Libération :

Christelle, 26 ans, veut témoigner que ces questions touchent l'ensemble des femmes des cités, et pas seulement les immigrées. "J'ai grandi dans une banlieue de la région parisienne. Avec ma tête de 'Française de souche' comme on nous appelle, nous aussi on est victimes de discrimination. Ça n'arrive pas qu'aux beurettes ou aux Blacks ». Même si elle tient beaucoup à cette précision, Fadela Amara, qui se dit musulmane pratiquante, le reconnaît: «Dans les familles maghrébines, les instruments d'oppression sont plus forts. Les courants fondamentalistes ont eu un effet direct sur les garçons : cela s'est immédiatement traduit par de la violence sur les sœurs, les voisines ${ }^{23}$.

Dans le discours d'information, les « jeunes filles des banlieues " sont en effet victimes, en plus du sexisme des garçons de leur âge, de la « culture » de leurs parents, culture nourrie par les traditions (mises en scène comme archaïques) corrélées à la religion musulmane. TF1 a par exemple fait témoigner une jeune femme d'origine turque victime d'un mariage forcé : «J'en pouvais plus, je... si je restais là-bas, c'est sûr que j'allais me suicider parce que j'peux plus vivre comme ça. Mes parents, ils m'laissaient rien faire, j'pouvais pas sortir, j'pouvais rien faire en plus tous les jours ils me harcelaient de me marier! ${ }^{24}$. Les références à l'islam (nombreuses dans le corpus) ont fortement marqué l'attribut ethnoracial du stéréotype de la «jeune fille des banlieues». Le groupe pour qui NPNS s'est mobilisé a été mis en scène comme vivant dans une famille pratiquant la religion musulmane.

L'identité socio-discursive des sujets d'énonciation théorique interrogés par les journalistes a été construite, dans les médias d'information généraliste, comme étant propre à toutes les adolescentes et jeunes femmes d'origine maghrébine de nationalité française. Ce groupe, produit comme «réel» dans la sphère publique a été représenté comme étant intrinsèquement victime de violences de genre commises par le groupe des jeunes garçons à l'attribut ethnoracial marqué. Les femmes «blanches » qui subissent des agressions sexistes sont construites comme des exceptions, tandis que les femmes «nonblanches » sont mises en scène comme étant toutes soumises à l'oppression des hommes de leur entourage. 


\section{Conclusion}

Avec la médiatisation de NPNS, les journalistes des médias d'information généraliste ont ethnoracialisé et construit le problème des violences de genre comme étant « réel ». Le mouvement a ainsi été mis en scène comme le représentant des jeunes filles d'origine nord-africaine qui se mobilise pour dénoncer le sexisme des garçons à l'attribut ethnoracial marqué. En réponse à cette couverture médiatique, NPNS a été accusé de stigmatiser les jeunes hommes d'origine maghrébine à l'extérieur du champ médiatique ${ }^{25}$. Un contre-mouvement s'est même constitué pour mettre en cause les discours dépréciatifs portés par le collectif en direction des minorités ethnoraciales : Ni proxo ni macho. L'utilisation et la mise en valeur du discours rapporté a permis aux professionnels des médias de ne pas être mis en cause dans ces processus de stigmatisation ${ }^{26}$ accusations qui sont exprimées régulièrement en direction de la sphère médiatique depuis une vingtaine d'années (Boyer et Lochard, op.cit.) - alors que, par les choix de cadrage ou du discours rapporté, ils ont largement participé à la construction et à la réactivation des stéréotypes négatifs utilisés pour représenter les «non-blancs» à l'œuvre depuis une trentaine d'années.

\section{BIBLIOGRAPHIE}

Arendt H., (1994 [1961]), Condition de l'homme moderne, Paris, Calmann-Lévy, 414 p.

Austin J. L., (2002 [1970]), Quand dire c'est faire, Paris, Éditions du Seuil, 202 p.

Boyer H. et Lochard G., (1998), Scènes de télévision en banlieues 1950-1994, Paris, INA/L'Harmattan, $201 \mathrm{p}$.

Brekhus W., (2005), Une sociologie de l'« invisibilité » : réorienter notre regard, in Réseaux, n 129-130, pp. 243-272.

Charaudeau P., (2005), Les médias et l'information. L'impossible transparence du discours, Bruxelles, INA/De Boeck, 250 p.

De Rudder V., Poiret C. et Vourc'h F., (2000), L'inégalité raciste. L'universalité républicaine à l'épreuve, Paris, PUF, $224 \mathrm{p}$.

Delforce B. et Noyer J., (1999), Pour une approche interdisciplinaire des phénomènes de médiatisation : constructivisme et discursivité sociale, in Études de Communication, $\mathrm{n}^{\circ} 22$, pp. 13-39.

Foucault M., (1976), Histoire de la sexualité 1. La volonté de savoir, Paris, Gallimard, 211 p.

Guénif-Souilamas N., (2003), Ni putes, ni soumises, ou très pute, très voilée ?, in Cosmopolitiques, $\mathrm{n}$ 4, pp. 53-65.

Hancock C., (2008), Décoloniser les représentations : esquisse d'une géographie culturelle de nos « Autres ", in Annales de géographie, $\mathrm{n}^{\circ}$ 660-661, pp. 116-128.

Jost F., (2003), La télévision du quotidien. Entre réalité et fiction, Bruxelles, De Boeck, 230 p. 
Komur G., (2004), L'îlot textuel et la prise de distance par le locuteur dans le genre journalistique, in Munoz J. M., Marnette S. et Rosier L. (éds.), Le discours rapporté dans tous ses états, Paris, L'Harmattan, pp. 54-63.

Krieg A., (1996), «La purification ethnique » dans la presse. Avènement et propagation d'une formule, in Mots, $n^{\circ}$ 47, pp. 109-125.

Laroche-Bouvy D., (1988), Émergence de l'interaction verbale dans la presse écrite : fonction de la citation, in Charaudeau P. (dir.), La presse, produit, production, réception, Paris, Éditions Didier Érudition, pp. 113-130.

Mills-Affif E., (2004), Filmer les immigrés. Les représentations audiovisuelles de l'immigration à la télévision française. 1960-1986, Bruxelles, De Boeck/INA, 299 p.

Molotch H. et Lester M., (1996), Informer : une conduite délibérée de l'usage stratégique des événements, in Réseaux, $\mathrm{n}^{\circ}$ 75, pp. 23-41.

Mortureux M.-F., (1993), Paradigmes désignationnels, in Semen, nº 8, pp. 117-136.

Neveu E., (1999), Médias, mouvements sociaux, espaces publics, in Réseaux, n 98, pp. 17-85.

Quéré L., (1982), Des miroirs équivoques. Aux origines de la communication moderne, Paris, Aubier Montaigne, $214 \mathrm{p}$.

Thompson J. B., (2005), La nouvelle visibilité, in Réseaux, n 129-130, pp. 59-87.

Voirol O., (2005), Les luttes pour la visibilité. Esquisse d'une problématique, in Réseaux, n 129-130, pp. 89-121.

\section{NOTES}

1. Sur les 33 articles de presse et 16 sujets de JT que compte notre corpus, 17 articles ont été publiés et 10 séquences de JT ont été diffusées autour du 8 mars 2003.

2. Le marquage social renvoie, selon Wayne Brekhus, aux "manières dont les acteurs sociaux perçoivent activement une des faces d'un contraste tout en ignorant l'autre face, conçue comme épistémologiquement non problématique» (Brekhus, 2005, 246). Pour faire référence à ce processus de marquage social, nous utiliserons les termes «non-blanc(s) » et «blanc(s) » qui renvoient, respectivement, aux individus qui sont marqués par leur attribut ethnoracial et à ceux qui ne le sont pas.

3. Voir les travaux d'Henri Boyer et de Guy Lochard (1998) et d'Edouard Mills-Affif (2004).

4. Cette analyse se concentre sur un point précis de l'étude plus vaste et exhaustive de la médiatisation de NPNS effectuée de janvier 2002 à décembre 2010 et réalisée dans le cadre de notre doctorat en sciences de l'information et de la communication.

5. John L. Austin (2002 [1970]) définit les énoncés performatifs comme des énoncés qui, en plus de « dire », accomplissent une action.

6. Le témoignage est un genre que nous avons créé. Il s'agit d'un article de presse ou d'une séquence de JT qui met en scène la parole d'individus sur leur vie quotidienne (ou sur un moment de leur vie), mais qui se réfère à l'intime.

7. Sur le processus d'ethnoracialisation, voir Véronique De Rudder et al. (2000).

8. Sur la fonction authentifiante du discours rapporté, voir Danielle Laroche-Bouvy (1988).

9. Le Monde (25/10/2002), « Pour les garçons, celle qui fume dans la rue ou qui n'est plus vierge, c'est une pute $»$, p. 11.

10. FRANCE 3 (14/02/2003), Soir 3. 
11. Nous avons effectué un relevé systématique au sein du corpus des syntagmes à valeur désignative utilisés pour désigner ce groupe («les jeunes filles des quartiers », « une femme », "une adolescente »...). Nous avons également opéré un classement des auteurs de ces syntagmes (journalistes, membres de NPNS...).

12. Le classement des militantes de NPNS au sein des catégories «blanches » et «non-blanches » a été effectué par elles-mêmes et les journalistes.

13. Fadela Amara, Safia Lebdi et Loubna Méliane ont été citées 66 fois alors que Christelle Raspolini et Ingrid Renaudin ont été mentionnées seulement 8 fois ( 4 fois chacune).

14. LibÉRATION (08/03/2003), « Mon frère m'a dit - 'ce que tu dis est juste' », pp. 18-19.

15. LibÉRATION (26/02/2003), « Soumission impossible », p. 36.

16. LE FIGARO (05/02/2003), « La rage au ventre », p. 33.

17. LE MONDE (25/10/2002), «Pour les garçons, celle qui fume dans la rue ou qui n'est plus vierge, c'est une pute », p. 11.

18. Libération (15/05/2002), « Femmes des cités, femmes révoltées », p. 19.

19. FRANCE $2(30 / 01 / 2003)$, JT de $20 \mathrm{~h}$.

20. LE FIGARO (05/02/2003), « La rage au ventre », p. 9.

21. France 3 (08/03/2003), JT de $20 \mathrm{~h}$.

22. LibÉRATION (08/03/2003), « Mon frère m'a dit - 'ce que tu dis est juste' ", pp. 18-19.

23. LiBÉRATION (31/01/2003), « La longue marche des femmes des cités », p. 17.

24. TF1 (06/03/2005), JT de $13 \mathrm{~h}$.

25. Cette mise en cause a été formulée par la sociologue Nacira Guénif-Souilamas (2003) ou encore par Houria Bouteldja, la porte parole du Mouvement des Indigènes de la République.

26. En plus d'authentifier les faits rapportés, la mise en scène d'une parole extérieure au journal permet aux journalistes de ne pas porter la responsabilité des propos tenus (Komur, 2004).

\section{RÉSUMÉS}

Les médias d'information généraliste, en plus de permettre aux mobilisations collectives d'accéder à la visibilité sociale, peuvent authentifier le problème de société formulé par les groupes protestataires. En valorisant le témoignage d'adolescentes «non-blanches » au sein de leurs productions, les journalistes ont construit la cause des violences de genre dans les banlieues, portée par Ni putes ni soumises à partir de janvier 2002, comme ayant une existence sociale. Ce processus d'authentification par les médias a conduit à légitimer le combat de Ni putes ni soumises auprès de l'opinion publique et de la sphère politique.

In addition to enabling collective mobilizations in order to reach social visibility, mainstream media can authenticate the particular social issue formulated by protest groups. In valuing the testimony of non-white female teenagers in their productions, journalists construct the causes of gender-related violence in French suburbs as argued by the Ni Putes ni Soumises (Neither Whores nor Submissive) movement from January 2002 onward as having a social existence. This authentication process leads to legitimizing the movement's fight in the eyes of public opinion and the political sphere. 
INDEX

Keywords : media, media coverage, social movements, performativity, ethnoracial minorities, $\mathrm{Ni}$ putes ni soumises, testimony

Mots-clés : médiatisation, médias, mouvements sociaux, performativité, minorités ethnoraciales, Ni putes ni soumises, témoignage

\section{AUTEUR}

\section{MARION DALIBERT}

GERIICO - Université Lille 3

Marion Dalibert est docteure en sciences de l'information et de la communication au laboratoire GERiiCO de l'université Lille 3. Ces recherches portent sur l'accès à l'espace public des minorités ethnoraciales. Elle analyse en particulier le rôle de l'identité collective de la Nation française dans les processus de médiatisation des groupes protestataires qui ont procédé à une lutte pour la visibilité en tant que « non-blancs ». Adresse électronique : marion.dalibert@gmail.com. 